\title{
Cooperative Shape Control of Particle Formations
}

\author{
Fumin Zhang
}

\begin{abstract}
The shape of a robot formation is key to performance in cooperative missions. Existing approaches of shape control require the formation dynamics be expressed in a unified coordinate frame. We present a shape theoretical approach which allows each robot, modeled as a particle, freedom to choose a coordinate frame to describe observed formation shape dynamics. Without knowing such coordinate frames of other robots, each robot is able to perform cooperative control. This is made possible by a class of gauge covariant cooperative control laws to control position, orientation, and shape of the formation. The formation dynamics are modeled as controlled Lagrangian systems on Jacobi shape space, we use measurements of shape variables and angular velocity as feedback to control the shape and to stop the rotation of the entire formation. Using Lyapunov functions, we prove that controlled dynamics converge to an invariant set where desired shape is achieved. Our method allows the robots to use direct observations from their onboard sensors to stabilize the formation.
\end{abstract}

\section{INTRODUCTION}

Recent developments in robotic research have enabled cooperative missions performed by multiple mobile robots. Different robots can be coordinated to sample the ocean [1], to carry heavy load [2], and to safeguard buildings [3]. The geometric shape formed by the positions of the robots are coupled to the effectiveness of the sensing or actuation performance. Distributed Motion control and planning is required to achieve desired shape.

We model robots in a formation as particles subjected to control forces, and model the entire formation as a deformable body. The shape of a deformable body is invariant under translation and rotation, and is also independent of the coordinate system in which we choose to describe it. This shape information can be described by using the Jacobi shape variables ( [4]-[6]).

It is common practice to study the dynamics of a deformable body in an attached coordinate frame, called the body frame. In this frame, the controlled dynamics of the formation are derived using the Lagrange-D'Alambert principle. In the resulting equations, we combine control forces of all particles into controls for the shape, the position, and the orientation respectively. We aim to design a Lyapunov function based cooperative control law to stabilize a given shape and to stop the rotation of the entire formation.

A phenomena, which does not happen when a rigid body is in question, is that shape changes affect the orientation of the body in the inertial frame. A popular example is the falling cat problem. Shape changes also raise extra caution on how the body frame is established because when we change

F. Zhang is with School of Electrical and Computer Engineering, Georgia Institute of Technology, Savannah, GA 31407. Email: fumin@ece.gatech.edu. the body frame from one to another, some quantities are transformed in complex, shape-dependent ways. We want to allow each particle the freedom to attach its own choice of body coordinate frame to study the whole formation. This requirement often arises when certain coordinate system is more convenient than others towards getting more accurate estimates from sensory data. For this purpose, we search for a class of mechanic quantities that do not depend on the choice of body frame. We say these objects are either gauge-invariant or gauge-covariant. We show that freedom in the choice of body frames can be achieved by utilizing gauge invariant and gauge covariant objects to design the cooperative control law.

Section II serves as a brief introduction to Jacobi shape space. In section III, we formally establish the notions of gauge invariance and gauge covariance. To help understand the implication of these notions, and as a simple application of the Jacobi shape theory, we analyze geometric properties of a sequential pursuit problem in section IV. We discover that the gauge covariant angular velocity is conserved by such a system. In section $\mathrm{V}$, we introduce the gauge covariant cooperative control form. We show that combined control laws can be computed by each robot independently if gauge covariant cooperative control forms are used. In section VI, we design a gauge covariant control law which stabilizes desired shape with fixed orientation. Simulation results are presented in section VII.

\section{JACOBI SHAPE SPACE}

In the inertial coordinate frame, let $q_{i} \in \mathscr{R}^{3}$, where $i=$ $1,2, \ldots, N$, denote the coordinates of $N$ particles with mass $m_{i}$. The kinetic energy of this cluster is $K^{t o t}=\frac{1}{2} \sum_{i=1}^{N} m_{i}\left\|\dot{q}_{i}\right\|^{2}$. This kinetic energy is translation invariant. Let $M=\sum_{i=1}^{N} m_{i}$, and the center of mass is $q_{c}=\frac{\sum_{i=1}^{N} m_{i} q_{i}}{M}$. We seek $(N-1)$ independent vectors $\left(\rho_{f i}, i=1,2, \ldots, N-1\right)$ such that the kinetic energy has the separated form

$$
K^{t o t}=\frac{1}{2} M\left\|\dot{q}_{c}\right\|^{2}+\frac{1}{2} \sum_{i=1}^{N-1}\left\|\dot{\rho}_{f i}\right\|^{2} .
$$

Such a set of $\rho_{f i}$ are called Jacobi coordinates or Jacobi vectors. Jacobi coordinates are not unique. Methods of constructing Jacobi coordinates are discussed in our earlier work [7].

Let $Q=\mathscr{R}^{3 N}$ be the total configuration space of a formation of $N$ particles in 3D space. The space of Jacobi coordinates is $\mathscr{R}^{3 N-3}$. A rotation group $\mathbf{G}=S O(3)$ acts on $\mathscr{R}^{3 N-3}$ to the left: $\Phi_{g}\left(\rho_{f i}\right)=g \rho_{f i}$ for $g \in \mathbf{G}$. This action is proper and free except for the shapes where all $\rho_{f i}$ are collinear, i.e., all particles are on a straight line. We let the set $F_{0}$ be the set of 
all the Jacobi coordinates corresponding to collinear shapes. Let $F=\mathscr{R}^{3 N-3}-F_{0}$ and call it the Jacobi pre-shape space. It is an open submanifold of the configuration space. Since $\mathbf{G}$ acts properly and freely on $F$, the base space $B=F / \mathbf{G}$ is a smooth manifold and the canonical projection $\pi: F \rightarrow B$ is differentiable. $B$ is called the Jacobi shape space.

In dropping from $F$ to $B$, we get rid of the rotational symmetry from the Jacobi coordinates. After the reduction, the dimension of the shape space $B$ is $(3 N-6)$. On this shape space we can define shape coordinates $s_{j}$ for $j=1,2, \ldots(3 N-6)$ which are rigid motion invariant. Candidates for $s_{j}$ are functions of dot products $\left(\rho_{f i} \cdot \rho_{f j}\right)$ and triple products $\left(\rho_{f i} \cdot\left(\rho_{f j} \times \rho_{f k}\right)\right)$. Thus, mutual distances, mutual angles, areas and volumes formed by the line segments connecting the particles all serve as candidates for shape variables. There is a large statistical literature on the subject of shape space and shape coordinates( [8]-[10]).

One can establish a body coordinate system on a formation with certain shape. Then the orientation of this formation in the inertial frame can be described by an element $g \in S O(3)$. The Jacobi coordinates in these two coordinate systems have the following relationship: $\rho_{f i}=g \rho_{i}(\mathbf{s})$ where $\mathbf{s}=\left(s_{1}, s_{2}, \ldots, s_{3 N-6}\right)^{T}$. The $\rho_{i}$ are Jacobi coordinates in the body frame. They are vector valued functions of the shape variables $\mathbf{s}$ only.

Some mechanic quantities can be defined in the body frame. We define $\Omega$ to be the angular velocity which satisfies $\dot{g}=g \widehat{\Omega}$ where $\widehat{\Omega}$ is the $3 \times 3$ skew symmetric matrix created from $\Omega$ such that $\Omega \times \mathbf{x}=\widehat{\Omega} \mathbf{x}$ for any $3 \mathrm{D}$ vector $\mathbf{x}$. Let $\mathbf{e}$ be the $3 \times 3$ identity matrix, we define

$$
I(\mathbf{s})=\sum_{i=1}^{N-1}\left(\left\|\rho_{i}\right\|^{2} \mathbf{e}-\rho_{i} \rho_{i}^{T}\right)
$$

as the locked inertia tensor of the formation in the body coordinate frame. We also define

$$
A_{j}(\mathbf{s})=I^{-1} \sum_{i=1}^{N-1} \rho_{i} \times \frac{\partial \rho_{i}}{\partial s_{j}}
$$

as the vector potential functions. These quantities are defined on the shape space, i.e. they are independent of orientation, because $\rho_{i}$ only depend on shape coordinates. We refer to [11] for a geometrically intrinsic approach to formulate the shape space and associated mechanic quantities.

\section{Gauge Transform}

As discussed in the previous section, for a given shape $\mathbf{s}$, one can establish a body coordinate system and obtain a group element in $S O(3)$ which describes the orientation of the body. When the shape of the formation changes, the body coordinate system should be consistent. We require the procedure to establish a body coordinate system be shape independent. Such a shape independent procedure for establishing body coordinate system is called a gauge convention. Formally, a gauge convention is a diffeomorphism between $F$ and $G \times B$ mapping any point $\boldsymbol{\rho} \in F$ to $(g, \mathbf{s}) \in G \times B$ s.t. $g_{1} \boldsymbol{\rho} \mapsto\left(g_{1} g, \mathbf{s}\right)$ for all $g_{1} \in G$. For example, let $\left(q_{1}, q_{2}, q_{3}\right)$ describe three

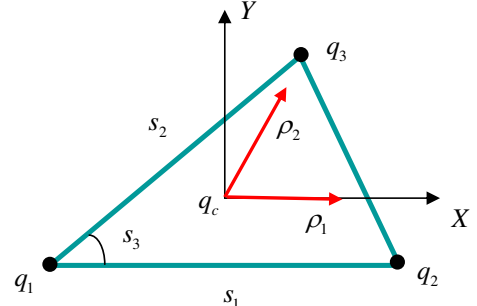

Fig. 1. One gauge convention for a triangular formation (Gauge A). The shape variable $s_{1}$ is the distance between particle 1 and $2, s_{2}$ is the distance between particle 1 and 3 , and $s_{3}$ is the angle measured counter-clockwise from $q_{1} q_{2}$ to $q_{1} q_{3}$

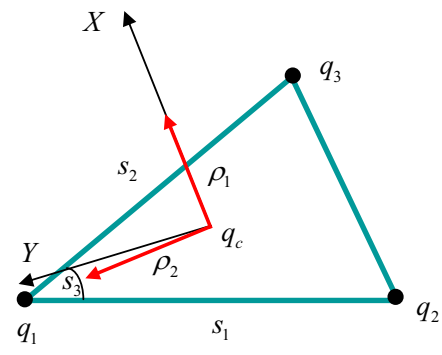

Fig. 2. Another gauge convention for a triangular formation (Gauge B). The shape variables are the same as in Gauge A. The Jacobi vectors are chosen differently from Gauge A.

points in $\mathscr{R}^{3}$. Except for the singular cases of collinear shapes, one can establish the body coordinate system of this triangular formation, shown in Figure 1, as follows:

1) Place the origin at center of mass, let the $x$-axis be aligned with vector $q_{1} q_{2}$.

2) Let the $y$-axis be in the plane formed by triangle $q_{1} q_{2} q_{3}$, be perpendicular to the $x$-axis, and point towards point $q_{3}$.

3) Let the $z$-axis form a right handed coordinate system with $x$-axis and $y$-axis.

This procedure is shape independent ,i.e., it does not depend on the distances between the three particles or the area of the triangular formation. We call this gauge convention Gauge A.

Gauge conventions are not unique. We show another gauge convention, which we call Gauge B, in Figure 2. Gauge B is established as follows:

1) Place the origin at center of mass, let the $x$-axis be aligned with vector $q_{2} q_{3}$.

2) Let the $y$-axis be in the plane formed by triangle $q_{1} q_{2} q_{3}$, be perpendicular to the $x$-axis and point towards point $q_{1}$.

3) Let the $z$-axis form a right handed coordinate system with $x$-axis and $y$-axis.

Let $g \in S O(3)$ describes the orientation of the formation for any shape $\mathbf{s}$ under one gauge convention. Let $g_{1} \in S O(3)$ describes the orientation of the formation for the same $\mathbf{s}$ under another gauge convention. Then by the property of $S O(3)$, there exists $h(\mathbf{s})$ such that $g=g_{1} h^{T}(\mathbf{s})$ where $h: B \rightarrow S O(3)$ 
is a $S O(3)$ valued function on $B$. This right action of $h(\mathbf{s})$ on $S O(3)$ is called a gauge transform. Because a gauge transform is a shape dependent group action, an object which obeys simple transformation rules under rigid group action by $S O(3)$ may violate such rules under a gauge transform. We say an object is gauge invariant if it is invariant under any gauge transform. We say an object is gauge covariant if it obeys the transformation rules for rigid group action by $S O(3)$ when it is subjected to a gauge transform. An example of a gauge invariant object is the collection of shape variables $\mathbf{s}$. An example of a gauge covariant object is a Jacobi vector $\rho_{i}$. Because $g \rho_{i}=\rho_{f i}=g_{1} \rho_{i 1}$, this implies that $\rho_{i}=h(\mathbf{s}) \rho_{i 1}$. Hence $\rho_{i 1}$ is transformed to $\rho_{i}$ via the left action by $h(\mathbf{s})$ by the same rule as if $h$ does not depend on $\mathbf{s}$.

The angular velocity $\Omega$ is not gauge covariant. We define a combined angular velocity as $\Upsilon=\Omega+\sum_{j=1}^{3 N-6} A_{j} \dot{s}_{j}$. We proved in [12] the following lemma:

Lemma 3.1: $\Upsilon$ is gauge covariant, i.e. $\Upsilon=h(s) \Upsilon_{1}$ under the gauge transform $g=g_{1} h^{T}(\mathbf{s})$.

Thus, $\Upsilon$ can be viewed as the gauge covariant angular velocity.

\section{Example: Analysis of Sequential Pursuit}

In this section we apply shape theory to analyze the problem of sequential pursuit, also called the "dog chasing" problem. The simplest such problem is of the following form: Let $\left(q_{1}, q_{2}, q_{3}\right)$ denote the positions of three particles in $\mathscr{R}^{3}$. Suppose they satisfy the equations

$$
\dot{q}_{1}=q_{2}-q_{1} ; \dot{q}_{2}=q_{3}-q_{2} ; \dot{q}_{3}=q_{1}-q_{3} .
$$

We want to show that from any initial configuration, the three particles converge to the centroid of the initial shape. The solution was known to Brocard in 1877 [13]. It was studied later in [14] and more recently in [15]. From a shape theoretic point view, we obtain some new insights to this classical problem.

The proof for convergence is straightforward after we express the system equations (4) using Jacobi vectors. It is true that $\dot{q}_{c}=\frac{1}{3}\left(\dot{q}_{1}+\dot{q}_{2}+\dot{q}_{3}\right)=0$. One way of defining the Jacobi vectors is

$$
\rho_{f 1}=\frac{1}{\sqrt{2}}\left(q_{2}-q_{1}\right) ; \rho_{f 2}=\sqrt{\frac{2}{3}}\left(q_{3}-\frac{q_{2}+q_{1}}{2}\right),
$$

and the inverse transform is

$$
\begin{aligned}
& q_{1}=q_{c}-\frac{\sqrt{2}}{2} \rho_{f 1}-\frac{1}{\sqrt{6}} \rho_{f 2} \\
& q_{2}=q_{c}+\frac{\sqrt{2}}{2} \rho_{f 1}-\frac{1}{\sqrt{6}} \rho_{f 2} \\
& q_{3}=q_{c}+\frac{\sqrt{2}}{\sqrt{3}} \rho_{f 2} .
\end{aligned}
$$

Then the pursuit equations in Jacobi coordinates are

$$
\dot{\rho}_{f 1}=-\frac{3}{2} \rho_{f 1}+\frac{\sqrt{3}}{2} \rho_{f 2} ; \dot{\rho}_{f 2}=-\frac{\sqrt{3}}{2} \rho_{f 1}-\frac{3}{2} \rho_{f 2} .
$$

This system is linear with eigenvalues equal to $-\frac{3}{2} \pm \mathbf{i} \frac{\sqrt{3}}{2}$. Therefore, as $t \rightarrow \infty, \rho_{f 1}$ and $\rho_{f 2} \rightarrow 0$. Since $q_{c}$ is fixed, the three particles will converge to $q_{c}$.

By using shape theory, we analyze the geometric properties of the convergence for the triangular shape. We compute how the shape of the deformable body formed by the three particles evolves in time and how fast the whole body is rotating around the centroid.

We use Gauge A as shown in Figure 1 to analyze the deformable body. Three shape variables are chosen: $s_{1}$ is the distance between particles 1 and $2, s_{2}$ is the distance between particles 1 and 3, and $s_{3}$ is the angle in Figure 1. These three variables are enough to describe a triangle because the dimension of the Jacobi shape space formed by three particles is 3 .

For simplicity, we assume that $m_{1}=m_{2}=m_{3}=1$. Under the Gauge A, the vector $\left(q_{2}-q_{1}\right)$ is $\left(s_{1}, 0,0\right)^{T}$, and the vector $\left(q_{3}-\frac{q_{2}+q_{1}}{2}\right)$ is $\left(s_{2} \cos \left(s_{3}\right), s_{2} \sin \left(s_{3}\right), 0\right)^{T}$. Thus, the Jacobi vectors in the body frame are

$$
\begin{aligned}
& \rho_{1}=\sqrt{\mu_{1}}\left(s_{1}, 0,0\right)^{T} \\
& \rho_{2}=\sqrt{\mu_{2}}\left(s_{2} \cos \left(s_{3}\right)-\mu_{1} s_{1}, s_{2} \sin \left(s_{3}\right), 0\right)^{T}
\end{aligned}
$$

where $\mu_{1}=\frac{1}{2}$ and $\mu_{2}=\frac{2}{3}$. We can calculate the locked inertia tensor $I$ using (2) i.e.

$$
I=\left\|\rho_{1}\right\|^{2} \mathbf{e}-\rho_{1} \rho_{1}^{T}+\left\|\rho_{2}\right\|^{2} \mathbf{e}-\rho_{2} \rho_{2}^{T} .
$$

The results, element by element, are

$$
\begin{aligned}
& I_{11}=\mu_{2} s_{2}^{2} \sin ^{2}\left(s_{3}\right) \\
& I_{12}=I_{21}=-\mu_{2} s_{2} \sin \left(s_{3}\right)\left(s_{2} \cos \left(s_{3}\right)-\mu_{1} s_{1}\right) \\
& I_{22}=\mu_{1} s_{1}^{2}+\mu_{2}\left(s_{2} \cos \left(s_{3}\right)-\mu_{1} s_{1}\right)^{2} \\
& I_{33}=\mu_{1} s_{1}^{2}+\mu_{2}\left(\left(s_{2} \cos \left(s_{3}\right)-\mu_{1} s_{1}\right)^{2}+s_{2}^{2} \sin ^{2}\left(s_{3}\right)\right) \\
& I_{23}=I_{32}=I_{13}=I_{31}=0 .
\end{aligned}
$$

In order to calculate the gauge potential $A$, we use (3), i.e.

$$
A_{j}=I^{-1}\left(\rho_{1} \times \frac{\partial \rho_{1}}{\partial s_{j}}+\rho_{2} \times \frac{\partial \rho_{2}}{\partial s_{j}}\right)
$$

for $j=1,2,3$. The results are

$$
\begin{aligned}
& A_{1}=\mu_{2} \frac{1}{I_{33}}\left[0,0, \mu_{1} s_{2} \sin \left(s_{3}\right)\right]^{T} \\
& A_{2}=\mu_{2} \frac{1}{I_{33}}\left[0,0,-\mu_{1} s_{1} \sin \left(s_{3}\right)\right]^{T} \\
& A_{3}=\mu_{2} \frac{1}{I_{33}}\left[0,0, s_{2}^{2}-\mu_{1} s_{1} s_{2} \cos \left(s_{3}\right)\right]^{T} .
\end{aligned}
$$

We want to find $\Omega$, the rotation speed of the body and $\dot{s}_{i}$ for $i=1,2,3$. There are altogether six unknowns. These unknowns can be solved from the six equations given by

$$
\dot{\rho}_{j}=\Omega \times \rho_{j}+\sum_{i=1}^{3} \frac{\partial \rho_{j}}{\partial s_{i}} \dot{s}_{i}
$$

for $j=1,2$ c.f. [7], [12]. Let $\Omega=\left[\Omega_{x}, \Omega_{y}, \Omega_{z}\right]^{T}$. We can get the following results: $\dot{s}_{1}=-2 s_{1}+s_{2} \cos \left(s_{3}\right), \dot{s}_{2}=-s_{2}-s_{1} \cos \left(s_{3}\right)$, $\dot{s}_{3}=\left(\frac{s_{1}}{s_{2}}-\frac{s_{2}}{s_{1}}\right) \sin \left(s_{3}\right), \Omega_{x}=0, \Omega_{y}=0$, and $\Omega_{z}=\frac{s_{2}}{s_{1}} \sin \left(s_{3}\right)$. 
We let $S_{1}=s_{1}^{2}+s_{2}^{2}+\left(s_{1}^{2}+s_{2}^{2}-2 s_{1} s_{2} \cos \left(s_{3}\right)\right)$ and $S_{2}=$ $\frac{1}{2} s_{1} s_{2} \sin \left(s_{3}\right)$. We call $\sqrt{S_{1}}$ the size of the formation because $S_{1}$ is the sum of the square length of the three edges. $S_{2}$ measures the area of the triangle. Then by direct calculation one can verify that $\dot{S}_{1}=-3 S_{1}$ and $\dot{S}_{2}=-3 S_{2}$.

We now show that the gauge covariant angular velocity $\Upsilon=$ $\Omega+\sum_{i=1}^{3} A_{i} \dot{s}_{i}$ is a constant vector. From equations (12) and the fact that $\Omega_{x}=\Omega_{y}=0$, we conclude that $\Upsilon_{x}=\Upsilon_{y}=0$. Then one can verify that

$$
\Upsilon_{z}=\Omega_{z}+A_{1 z} \dot{s}_{1}+A_{2 z} \dot{s}_{2}+A_{3 z} \dot{s}_{3}=6 \frac{S_{2}}{S_{1}} .
$$

This means that the rotational speed is six times the proportion between the area and the square size of the formation. We can calculate the derivative of $\Upsilon_{z}$ as

$$
\dot{\Upsilon}_{z}=6 \frac{S_{1} \dot{S}_{2}-S_{2} \dot{S}_{1}}{S_{1}^{2}}=0 .
$$

This proves that $\Upsilon$ is a constant.

\section{Controlled Lagrange equations And Gauge COVARIANT CONTROL LAWS}

Let $\mathbf{A}=\left[A_{1}, A_{2}, \ldots, A_{3 N-6}\right]$. Then we can rewrite the total kinetic energy in block diagonalized form as

$$
\begin{aligned}
K^{t o t} & =\frac{1}{2} M\left\|\dot{q}_{c}\right\|^{2}+\frac{1}{2}(\Omega+\mathbf{A} \dot{\mathbf{s}})^{T} I(\Omega+\mathbf{A} \dot{\mathbf{s}})+\frac{1}{2} \dot{\mathbf{s}}^{T} G \dot{\mathbf{s}} \\
& =\frac{1}{2} M\left\|\dot{q}_{c}\right\|^{2}+\frac{1}{2} \Upsilon^{T} I \Upsilon+\frac{1}{2} \dot{\mathbf{s}}^{T} G \dot{\mathbf{s}}
\end{aligned}
$$

where elements of $G$ are

$$
G_{j k}=-A_{j}^{T} I A_{k}+\sum_{i=1}^{N-1} \frac{\partial \rho_{i}^{T}}{\partial s^{j}} \frac{\partial \rho_{i}}{\partial s^{k}} .
$$

The Lagrangian is $K^{t o t}-V$ where $V$ is a potential function on shape space.

In [16] and [17], the Lagrange-D'Alembert principle for rigid body dynamics is rigorously formulated. We apply such results and the resulting controlled Lagrange equations are

$$
\begin{gathered}
M \ddot{q}_{c}=-\frac{\partial V}{\partial q_{c}}+u_{c} \\
\frac{d}{d t}(I)=-\Omega \times I-g^{-1} \frac{\partial V}{\partial g}+u_{g}, \\
\frac{d}{d t}(G \dot{\mathbf{s}})+\mathbf{A}^{T} \frac{d}{d t}(\Upsilon) \\
=\frac{1}{2}\left[\frac{\partial I}{\partial \mathbf{s}}\right]^{*}:(\Upsilon, \Upsilon)+\left(\left[\frac{\partial \mathbf{A}}{\partial \mathbf{s}}\right]^{*}-\left[\frac{\partial \mathbf{A}}{\partial \mathbf{s}}\right]\right)[\dot{\mathbf{s}}, I] \\
+\frac{1}{2}\left[\frac{\partial G}{\partial \mathbf{s}}\right]^{*}(\dot{\mathbf{s}}, \dot{\mathbf{s}})-\frac{\partial V}{\partial \mathbf{s}}+u_{s} .
\end{gathered}
$$

In these equations, $\left[\frac{\partial \mathbf{A}}{\partial \mathbf{s}}\right],\left[\frac{\partial I}{\partial \mathbf{s}}\right]$, and $\left[\frac{\partial G}{\partial \mathbf{s}}\right]$ are third order tensors obtained by taking the Frechet derivatives of the matrices $\mathbf{A}$, $I$, and $G$ with respect to vector $\mathbf{s}$, and $\left[\frac{\partial \mathbf{A}}{\partial \mathbf{s}}\right]^{*},\left[\frac{\partial I}{\partial \mathbf{s}}\right]^{*}$, and $\left[\frac{\partial G}{\partial \mathbf{s}}\right]^{*}$ are the cyclic transpose of these third order tensors c.f. [16] and [18].
The Lagrange equations for the formation in the lab coordinate system are simply the set of Newton's equations:

$$
\ddot{q}_{i}=\mathbf{f}_{i}
$$

for $i=1,2, \ldots, N$. We have established the relationship between the control forces $\mathbf{f}_{i}$ and $\left(u_{c}, u_{g}, u_{s}\right)$ in [12].

Although controllers can be designed by computing $\left(u_{g}, u_{s}\right)$ first and then find out $\mathbf{f}_{i}$, which is the actual force on each particle, this procedure is gauge dependent. This posts a constraint that each particle has to agree on the same gauge convention. Therefore, they have to agree on the measurement of gauge dependent quantities such as $\Omega$. This implies that some particles have to estimate the gauge dependent quantities indirectly from its observations. Such indirect estimates may introduce more severe noise than direct observations. For example, in the case of a three particle formation, suppose each particle is able to measure the relative positions between itself and the other two particle. If all three particles agree on Gauge A in section IV, then particle 3 has to estimate $q_{1} \vec{q}_{2}$ to determine the $x$-axis of the body coordinate system. This estimate incorporates noise from observing both particle 1 and particle 2.

We show that by transforming $\left(u_{g}, u_{s}\right)$ into a proper gauge covariant form, we establish a controller design procedure which allows each particle to use its own gauge convention. In our example above, for particle 3, we may use Gauge B where the $x$-axis of the body coordinate system is aligned with the vector $q_{2} q_{3}$, which is observed directly by sensors of particle 3.

We define the gauge covariant cooperative control form to be the pair $\left(\alpha_{g}, \alpha_{s}\right)$ where $\alpha_{g}$ and the $k$ th component of $\alpha_{s}$ satisfy

$$
\begin{aligned}
\alpha_{g} & =u_{g} \\
\alpha_{s k} & =u_{s k}-<u_{g}, A_{k}>
\end{aligned}
$$

for $k=1,2, \ldots, 3 N-6$.

This cooperative control form allows us to design $\left(\alpha_{g}, \alpha_{s}\right)$ as gauge covariant quantities. Then the actual forces $\mathbf{f}_{j}$ can be computed by each member of the formation in its own coordinate system with its own choice of Jacobi vectors.

Theorem 5.1: Let particle 1 and particle 2 be two members of a $N$-particle formation governed by the controlled Lagrange equations (21),(18),(19) and (20). Let $\rho_{f j}^{1}$ and $\rho_{f j}^{2}$ where $j=1,2, \ldots,(N-1)$ denote the two different sets of Jacobi vectors chosen by the two particles. The two particles have established two gauge conventions for the formation that are connected by the gauge transform $g^{1}=g^{2} h^{T}(\mathbf{s})$ for each orientation of the formation. Suppose the feedback control $\left(\alpha_{g}^{1}, \alpha_{s}^{1}\right)$ computed by particle 1 and $\left(\alpha_{g}^{2}, \alpha_{s}^{2}\right)$ computed by particle 2 are functions of gauge invariant and gauge covariant quantities, i.e. $\left(\alpha_{g}^{1}, \alpha_{s}^{1}\right)=\left(h(\mathbf{s}) \alpha_{g}^{2}, \alpha_{s}^{2}\right)$. Suppose the two particles agree on the same set of shape variables $s_{k}$ for $k=1,2, \ldots,(3 N-6)$ and use the same control force $u_{c}$ for the center of the formation. Then the forces applied to each particle computed by particle 1 are identical to the forces for 
each particle computed by particle 2 when compared in the inertial frame i.e. $\mathbf{f}_{i}^{1}=\mathbf{f}_{i}^{2}$ for $i=1,2, \ldots N$.

We provided a detailed proof in [12].

\section{GAUGE COVARIANT FEEDBACK CONTROL USING SHAPE MEASUREMENTS}

Suppose the potential function $V$ is invariant under translation and rotation i.e. $V$ is only a function of the shape variables. We can define a function on the tangent bundle of the preshape space as

$$
V_{L}=\frac{1}{2}\left\|\mathbf{s}-\mathbf{s}^{0}\right\|^{2}+\frac{1}{2}(\Omega+\mathbf{A} \dot{\mathbf{s}})^{T} I(\Omega+\mathbf{A} \dot{\mathbf{s}})+\frac{1}{2} \dot{\mathbf{s}}^{T} G \dot{\mathbf{s}}
$$

where $\mathbf{s}^{0}$ specifies a desired shape. The derivative of this function along the reduced dynamics (19) and (20) is

$$
\begin{aligned}
\dot{V}_{L} & =<\left(\mathbf{s}-\mathbf{s}^{0}\right), \dot{\mathbf{s}}>+<\Omega, u_{g}>+<\dot{\mathbf{s}}, u_{s}-\frac{\partial V}{\partial \mathbf{s}}> \\
& =<\dot{\mathbf{s}}, u_{s}-\frac{\partial V}{\partial \mathbf{s}}+\left(\mathbf{s}-\mathbf{s}^{0}\right)>+<\Omega, u_{g}>
\end{aligned}
$$

where we use $<$, $>$ for inner product.

We design a cooperative control law to be

$$
\begin{aligned}
& \alpha_{g}=-k_{1} \Upsilon \\
& \alpha_{s}=\frac{\partial V}{\partial \mathbf{s}}-\left(\mathbf{s}-\mathbf{s}^{0}\right)-k_{1} \dot{\mathbf{s}}
\end{aligned}
$$

where $k_{1}>0$ is a constant gain. This control law is gauge covariant. The resulting $\left(u_{g}, u_{s}\right)$ is

$$
\begin{aligned}
& u_{g}=-k_{1}(\Omega+\mathbf{A} \dot{\mathbf{s}}) \\
& u_{s}=\frac{\partial V}{\partial \mathbf{s}}-\left(\mathbf{s}-\mathbf{s}^{0}\right)-k_{1} \dot{\mathbf{s}}-k_{1} \mathbf{A}^{T}(\Omega+\mathbf{A} \dot{\mathbf{s}}) .
\end{aligned}
$$

Then, under such a control law, we have

$$
\dot{V}_{L}=-\|\dot{\mathbf{s}}\|^{2}-k_{1}\|\Omega+\mathbf{A} \dot{\mathbf{s}}\|^{2} \leq 0
$$

with $\dot{V}_{L}=0$ if and only if $\Omega=0$ and $\dot{\mathbf{s}}=0$

We apply LaSalle's invariance principle to argue that the controlled dynamics converge to the maximal invariant set $C_{1}$ within the set $M_{1}$ where $\dot{V}_{L}=0$. Hence

$$
M_{1}=\{(g, \mathbf{s}, \Omega, \dot{\mathbf{s}}) \in T F \mid \Omega=0, \dot{\mathbf{s}}=0\}
$$

and

$$
C_{1}=\left\{(g, \mathbf{s}, \Omega, \dot{\mathbf{s}}) \in M_{1} \mid \dot{\Omega}=0, \ddot{\mathbf{s}}=0\right\}
$$

In the system equations (19) and (20), letting $\Omega=0$ and $\dot{\mathbf{s}}=0$, we have $I \dot{\Omega}=u_{g}=0$ and $G \ddot{\mathbf{s}}+I \dot{\Omega}=u_{\mathbf{s}}-\frac{\partial V}{\partial \mathbf{s}}=\mathbf{s}-\mathbf{s}^{0}$. Thus on the set $C_{1}$, in order for $\dot{\Omega}=0$ and $\ddot{\mathbf{s}}=0$, we must have $\mathbf{s}-\mathbf{s}^{0}=0$. Therefore, we have proved the following theorem:

Theorem 6.1: Suppose the potential $V$ is rigid motion invariant. By using the cooperative feedback control law (25), the Jacobi shape $\mathbf{s}^{0}$ is locally asymptotically stabilized.
TABLE I

INITIAL STATES FOR THE THREE PARTICLES

\begin{tabular}{c|l|l}
\hline Particle & Position & Velocity \\
\hline 1 & $(0,0)$ & $(2,1)$ \\
\hline 2 & $(-2,-3.5)$ & $(-1,1)$ \\
\hline 3 & $(0.1,-0.5)$ & $(-1,1)$ \\
\hline
\end{tabular}

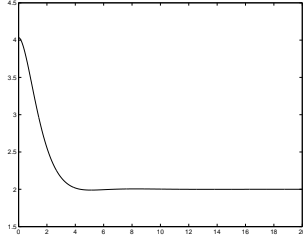

(a) changes in $s_{1}$

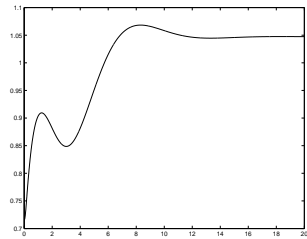

(c) changes in $s_{3}$

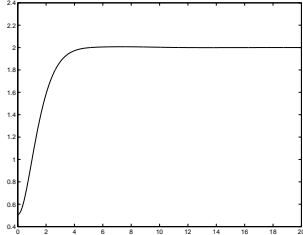

(b) changes in $s_{2}$

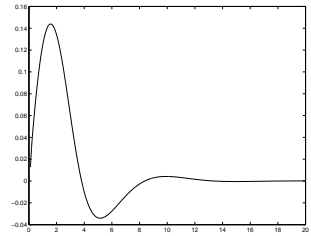

(d) Rotation speed
Fig. 3. Convergence of shape variables and rotation speed when all particles agree on the same gauge. These plots are identical to the case when particle 3 uses a different gauge.

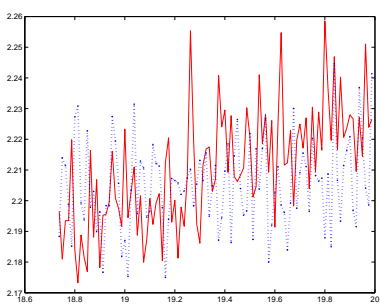

(a) Mean

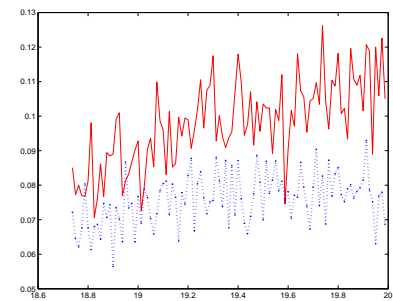

(b) Variance
Fig. 4. The mean and variance of $s_{1}$ in the last 5 seconds of simulation . Red solid line represents the case when all particles use the same gauge. Blue dotted line represents the case when particle 3 chooses a special gauge.

\section{Simulation Results}

We designed simulations to demonstrate the gauge covariant control law. We show here the example of a formation of three particles in the plane. Table I lists the initial positions and velocities of the three particles. Three shape variables are chosen as in section IV: $s_{1}$ is the distance between particle 1 and $2, s_{2}$ is the distance between particle 1 and 3 , and $s_{3}$ is the angle shown in Figure 1. The desired shape of the formation is an equilateral triangle determined by $s_{1}=s_{2}=2$ and $s_{3}=\frac{\pi}{3}$.

In experiment 1 , all particles use Gauge $\mathrm{A}$ in section IV. They also choose the same set of Jacobi vectors as in Figure 1. We assume no noise in the measurements of the shape variables. Under the gauge covariant control law (25), the formation converges to the desired shape. The convergence of the three shape variables are shown in Figure 3(a), 3(b) and $3(\mathrm{c})$. The rotation speed of the formation are shown in 


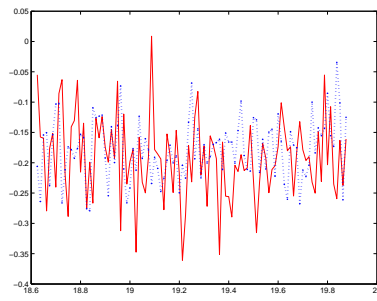

(a) Mean

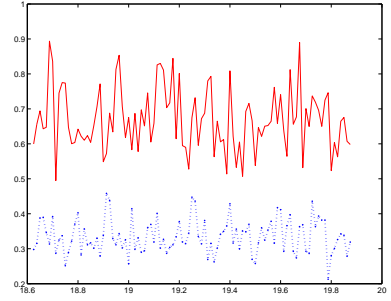

(b) Variance
Fig. 5. The mean and variance of the rotation speed in the last 5 seconds of the simulation. Red solid line represents the case when all particles use the same gauge. Blue dotted line represents the case when particle 3 chooses a special gauge.

Figure 3(d). We see that the formation stops rotating. On the other hand, the center of mass is moving constantly at velocity $(0,1)$.

In experiment 2, particle 1 and 2 still use Gauge A, but particle 3 uses Gauge B. Particle 3 also uses a new set of Jacobi vectors, shown in Figure 2, with

$$
\rho_{f 1}=\frac{1}{\sqrt{2}}\left(q_{3}-q_{2}\right) ; \rho_{f 2}=\sqrt{\frac{3}{2}}\left(q_{1}-\frac{q_{2}+q_{3}}{2}\right) .
$$

We use the same gauge covariant control law as in experiment 1. The formation also converges as shown in Figure 3. There is no difference between results in these two cases, as predicted by theorem 5.1 .

Next, we compare the performance of the gauge covariant control law when there are simulated noise in the shape measurements. In our simulation, each particle is able to determine the relative positions of the other two particles. We add independent Gaussian noise $\mathscr{N}(0,0.1)$ in the estimates for the relative positions, which introduce noise in the estimates for the shape variables and the rotational speed. If particle 3 uses the same gauge as particle 1 and 2, it has to estimate vector $q_{1} q_{2}$ in order to estimate the rotational speed. If particle 3 uses the special gauge as shown in Figure 2, it has to estimate $q_{2} q_{3}$. The estimate for $q_{1} q_{2}$ is derived from estimating both $q_{1} q_{3}$ and $q_{2} q_{3}$, hence more noisy than the estimate for $q_{2} q_{3}$.

To compare the results for the two cases, namely the case of same gauge convention and the case of different gauge conventions. We run 100 experiments in each case. We then compare the mean and variance of the values for the shape variables and the rotational speed in the last 5 seconds of the simulation when "steady state" is reached. As shown in Figure 4, using different gauge conventions outperforms using same gauge conventions in control of shape variable $s_{1}$, and similar results hold for $s_{2}$ and $s_{3}$. For the rotational speed, using different gauge conventions has a clear advantage. The variance of the rotational speed when same gauge conventions are used is much larger than the variance of the final rotational speed when different gauge conventions are used as shown in Figure 5.

\section{SUMMARY}

In this paper we have outlined an approach to design particle formation shape control based on Jacobi shape theory. We introduce a gauge covariant cooperative control form and demonstrate the advantage of using such form to reduce noise. A gauge covariant cooperative feedback law for locally stabilizing shape of interest is designed and theoretically justified.

\section{ACKNOWLEDGMENTS}

The author thanks Prof. P. S. Krishnaprasad for his guidance and collaboration on this work, and thanks Dr. E. W. Justh and Prof. N. E. Leonard for discussions and suggestions. This research was supported in part by NASA under NASA-GSFC Grant No. NAG5-10819, by the AFOSR under AFOSR Grant No. F49620-01-0415, by the ARO under ODDR\&E MURI97 Program Grant No. DAAG55-97-1-0114 to the Center for Dynamics and Control of Smart Structures (through Harvard University), and under ODDR\&E MURI01 Program Grant No. DAAD19-01-1-0465 to the Center for Communicating Networked Control Systems (through Boston University).

\section{REFERENCES}

[1] F. Zhang, D. M. Fratantoni, D. Paley, J. Lund, and N. E. Leonard, "Control of coordinated patterns for ocean sampling," International Journal of Control, vol. 80, no. 7, pp. 1186-1199, 2007.

[2] R. Gross, M. Bonani, F. Mondada, and M. Dorigo, "Autonomous selfassembly in swarm-bots," IEEE Trans. Robot., vol. 22, no. 6, pp. 1115 - 1130, 2006.

[3] J. Feddema, C. Lewis, and D. Schoenwald, "Decentralized control of cooperative robotic vehicles: theory and application," IEEE Trans. Robot. Automat., vol. 18, no. 5, pp. 852-864, 2002.

[4] C. G. J. Jacobi, Vorlesungen über Dynamik. Reimer, Berlin, 1866.

[5] V. Aquilanti and S. Cavalli, "Coordinates for molecular dynamics: Orthogonal local system," J. Chemical Physics, vol. 85, no. 3, pp. 13551361, 1986.

[6] R. Littlejohn and M. Reinsch, "Gauge fields in the separation of rotation and internal motions in the n-body problem," Reviews of Modern Physics, vol. 69, no. 1, pp. 213-275, 1997.

[7] F. Zhang, M. Goldgeier, and P. S. Krishnaprasad, "Control of small formations using shape coordinates," in Proc. of 2003 Internat. Conf. of Robotics and Automation. Taipei, Taiwan: IEEE, 2003, pp. 2510-2515.

[8] D. G. Kendall, "Shape manifolds, procrustean metrics, and complex projective spaces," Bulletin of London Math. Society, vol. 16, pp. 81$121,1984$.

[9] D. G. Kendall, D. Barden, T. Karne, and H. Le, Shape and shape theory. New York: Chichester, 1999.

[10] C. G. Small, The Statistical Theory of Shape. New York: Springer, 1996.

[11] R. Montgomery, "Optimal control of deformable bodies and its relation to gauge theory," in The Geometry of Hamiltonian Systems, T.Ratiu, Ed. MSRI Publications,Springer-Verlag, 1991, vol. 22.

[12] F. Zhang, "Geometric cooperative control of formations," Ph.D. dissertation, ISR Technical Report PHD2004-5, University of Maryland, 2004.

[13] H. Brocard, "Solution of Lucas's problem," Nouv. Corresp. Math., vol. 3, p. $280,1877$.

[14] A. Bernhart, "Polygons of pursuit," Scripta Mathematica, vol. 24, pp. 23-50, 1959.

[15] Z. Lin, M. Broucke, and B. Francis, "Local control strategies for groups of mobile autonomous agents," IEEE Transactions on Automatic Control, vol. 49, no. 4, pp. 622-629, 2004.

[16] L.-S. Wang, "Geometry,dynamics and control of coupled systems," Ph.D. dissertation, ISR Technical Report PhD90-5, University of Maryland, 1990.

[17] L.-S. Wang and P. S. Krishnaprasad, "Gyroscopic control and stabilization," Journal of Nonlinear Science, vol. 2, pp. 367-415, 1992.

[18] S. S. Antman, Nonlinear problems of elasticity. New York: SpringerVerlag, 1995. 